\title{
EM Cluster Analysis for Categorical Data
}

\author{
Jiří Grim \\ Institute of Information Theory and Automation \\ of the Czech Academy of Sciences, \\ P.O. Box 18, 18208 Prague 8, Czech Republic \\ grim@utia.cas.cz \\ http://ro.utia.cas.cz/mem.html
}

\begin{abstract}
Distribution mixtures with product components have been applied repeatedly to determine clusters in multivariate data. Unfortunately, for categorical variables the mixture parameters are not uniquely identifiable and therefore the result of cluster analysis may become questionable. We give a simple proof that any non-degenerate discrete product mixture can be equivalently described by infinitely many different parameter sets. Nevertheless a unique result of cluster analysis can be guaranteed by additional constraints. We propose a heuristic method of sequential estimation of components to guarantee a unique identification of clusters by means of EM algorithm. The application of the method is illustrated by a numerical example.
\end{abstract}

\section{Introduction}

The cluster analysis of categorical data is well known to be a difficult problem. Let us recall that arithmetical operations and therefore means and variances are undefined for categorical variables. Generally, the values of categorical variables are neither ordered nor there is any reasonable and commonly acceptable way to define a distance or similarity measure. Binary variables as a special case may appear to be naturally ordered but often there is no reliable argument to prefer one of the two possibilities to assign the values 0 and 1 . For these and other reasons the standard clustering algorithms are not directly applicable to multivariate categorical data.

One of the first statistical methods of cluster analysis of categorical data is due to Lazarsfeld 14. Motivated by sociological research he proposed fitting of multivariate Bernoulli mixtures to binary data to identify possible latent classes of respondents by means of mixture components. Wide application of the latent class (latent structure) analysis was enabled by the computationally efficient EM algorithm [4. Discussion of latent class analysis from a statistical point of view can be found in Fielding [5]. Other approaches to clustering and latent variable models are discussed e.g. by Vermunt et al. [18, (see also [1], 6], 15]).

\footnotetext{
* This research was supported by the EC project no. FP6-507752 MUSCLE, by the grant No. 1ET400750407 of the Grant Agency of the Academy of Sciences CR and partially by the project MŠMT 1M0572 DAR and GAČR 402/03/1310.
} 
Multivariate Bernoulli mixture is only a special case of the general conditional independence model which can be defined for general discrete variables (categorical, qualitative or nominal) as a finite mixture of product components. The application of discrete product mixtures to cluster analysis corresponds with the original approach of Lazarsfeld, however, in both cases there is a problem to justify the obtained solutions. The conditional independence model is not uniquely identifiable in case of categorical variables and therefore the result of cluster analysis becomes questionable.

In the present paper we first introduce the conditional independence model for unordered categorical variables and briefly describe the corresponding version of EM algorithm for estimation of mixtures (Sec. 2). Then we discuss the problem of identifiability of distribution mixtures with product components (Sec. 3) in connection with the recently considered concept of "practical identifiability" of multivariate Bernoulli mixtures [3. In Sec.4 we propose the method of sequential identification of mixture components as a tool to obtain unique clusters. The application of the method is illustrated by a numerical example. Finally we summarize the main results in the Conclusion.

\section{Conditional Independence Models}

Let $\xi_{1}, \ldots, \xi_{N}$ be a finite number of general discrete random variables. In particular, we assume that each variable $\xi_{n} \in \mathcal{X}_{n}$ takes on some categorical (nominal, qualitative) values from a finite set $\mathcal{X}_{n}$ without any type of ordering. Simultaneously, let $\mu$ be a discrete random variable taking on values from a finite set of integers $\mathcal{M}$

$$
P\{\mu=m\}=w_{m}, \quad m \in \mathcal{M}, \quad \sum_{m \in \mathcal{M}} w_{m}=1, \quad \mathcal{M}=\{1, \ldots, M\} .
$$

We suppose that the random variables $\xi_{n}$ are conditionally independent given the value of $\mu$. In other words we assume that the conditional probability distributions $F(\boldsymbol{x} \mid m), m \in \mathcal{M}$ of the random vector

$$
\boldsymbol{\xi}=\left(\xi_{1}, \ldots, \xi_{N}\right) \in \mathcal{X}, \quad \mathcal{X}=\mathcal{X}_{1} \times \ldots \times \mathcal{X}_{N}, \quad \mathcal{N}=\{1, \ldots, N\}
$$

can be expressed as a product of univariate conditional distributions $f_{n}\left(x_{n} \mid m\right)$ :

$$
P\{\boldsymbol{\xi}=\boldsymbol{x} \mid \mu=m\}=F(\boldsymbol{x} \mid m)=\prod_{n \in \mathcal{N}} f_{n}\left(x_{n} \mid m\right), \quad \boldsymbol{x} \in \mathcal{X}, \quad m \in \mathcal{M} .
$$

In view of Eqs. (1), (2) the unconditional joint probability distribution of the random vector $\boldsymbol{\xi}$ can be expressed in the form of a finite distribution mixture of product components:

$$
P(\boldsymbol{x})=\sum_{m \in \mathcal{M}} w_{m} F(\boldsymbol{x} \mid m)=\sum_{m \in \mathcal{M}} w_{m} \prod_{n \in \mathcal{N}} f_{n}\left(x_{n} \mid m\right), \quad \boldsymbol{x} \in \mathcal{X}, \quad\left(w_{m}>0\right) .
$$


Here the probability $w_{m}$ is usually called the weight of $m$-th component and $f_{n}\left(x_{n} \mid m\right)$ are the conditional (component specific) univariate distributions of the variables $\xi_{n}$ respectively. In this sense the distribution mixture (3) is defined by the parameter set $\Theta=\left\{M, w_{m}, f_{n}(\cdot \mid m), m \in \mathcal{M}\right\}$. 1

In case of dichotomous variables $\xi_{n} \in\{0,1\}$ the probability distribution (3) becomes the well known multivariate Bernoulli mixture

$$
P(\boldsymbol{x})=\sum_{m \in \mathcal{M}} w_{m} \prod_{n \in \mathcal{N}} \vartheta_{n m}^{x_{n}}\left(1-\vartheta_{n m}\right)^{1-x_{n}}, \quad \boldsymbol{x} \in\{0,1\}^{N}, \quad 0<\vartheta_{n m}<1
$$

which is a special case of the general conditional independence model (3).

The standard way to estimate the conditional independence models from data is to compute maximum-likelihood estimates of mixture parameters by means of the iterative EM algorithm [4, [13. In particular, let $\mathcal{S}$ be a set of independent observations of the random vector $\boldsymbol{\xi}$ :

$$
\mathcal{S}=\left\{\boldsymbol{x}^{(1)}, \boldsymbol{x}^{(2)}, \ldots, \boldsymbol{x}^{(K)}\right\}, \quad \boldsymbol{x}^{(k)} \in \mathcal{X}
$$

which are identically distributed with some unknown distribution mixture of the form (3). To compute the m.-l. estimates of the unknown parameters $w_{m}, f_{n}(\cdot \mid m)$ we maximize the likelihood function

$$
L=\frac{1}{|\mathcal{S}|} \sum_{x \in \mathcal{S}} \log P(\boldsymbol{x})=\frac{1}{|\mathcal{S}|} \sum_{x \in \mathcal{S}} \log \left[\sum_{m \in \mathcal{M}} w_{m} F(\boldsymbol{x} \mid m)\right]
$$

by means of the basic EM iteration equations

$$
\begin{aligned}
q(m \mid \boldsymbol{x}) & =\frac{w_{m} F(\boldsymbol{x} \mid m)}{\sum_{j \in \mathcal{M}} w_{j} F(\boldsymbol{x} \mid j)}, \quad w_{m}^{\prime}=\frac{1}{|\mathcal{S}|} \sum_{x \in \mathcal{S}} q(m \mid \boldsymbol{x}), \quad m \in \mathcal{M}, \\
f_{n}^{\prime}(\xi \mid m) & =\frac{1}{\sum_{x \in \mathcal{S}} q(m \mid \boldsymbol{x})} \sum_{x \in \mathcal{S}} \delta\left(\xi, x_{n}\right) q(m \mid \boldsymbol{x}), \quad \xi \in \mathcal{X}_{n}, \quad n \in \mathcal{N},
\end{aligned}
$$

where $w_{m}^{\prime}, f_{n}^{\prime}(\cdot \mid m)$ are the new parameter values and $\delta\left(\xi, x_{n}\right)$ denotes the usual delta-function, i.e. $\delta\left(\xi, x_{n}\right)=1$ for $\xi=x_{n}$ and otherwise $\delta\left(\xi, x_{n}\right)=0$.

The EM algorithm generates a nondecreasing sequence $\left\{L^{(t)}\right\}_{0}^{\infty}$. As the criterion (6) is bounded above $(L<0)$ the monotonic property implies convergence of the sequence $\left\{L^{(t)}\right\}_{0}^{\infty}$ to a possibly local maximum of the function (6) in the parameter space (for more details cf. e.g. [13]). Obviously, a local maximum may be starting-point dependent.

Given the estimated distribution mixture (3) we can characterize any data vector $\boldsymbol{x} \in \mathcal{X}$ by its affinity with the mixture components in terms of the conditional probabilities. The conditional posterior weights $q(m \mid \boldsymbol{x})$ are particularly useful if there is some interpretation of the mixture components, e.g. if the components correspond to some "latent classes" [14, "hidden causes" [15] or

\footnotetext{
${ }^{1}$ Here and in the following we assume that the parameter sets differing only by the order of components are identical.
} 
"clusters" having a specific meaning. This idea is closely related to the original latent structure analysis of Lazarsfeld.

There are also some other theoretical arguments justifying the finite distribution mixture (3) as a "latent class" model. It should be emphasized that the statistical relations among the random variables $\xi_{1}, \ldots, \xi_{N}$ are wholly explained by their dependence on the variable $\mu$ which is sometimes called the latent variable. Given the value of the latent variable $\mu$, the random variables $\xi_{n}$ are statistically independent, i.e. their interdependence is removed. In this sense the values of the variable $\mu$ can be viewed as "hidden causes" which cannot be observed directly but remove the statistical interaction between the observed variables $\xi_{1}, \ldots, \xi_{N}$. Once specified, the hidden cause $\mu$ would permit us to treat the visible variables $\xi_{n}$ in a simple way as if they were mutually independent [15. In view of these arguments the conditional independence model (3) is assumed to be "the most universal and distinctive characteristics featured by the notion of causality" (cf. [15], [16]).

Remark 1. It is easily verified that the conditional independence model (3) is not restrictive in the sense that any discrete probability distribution $P(\boldsymbol{x})$ on $\mathcal{X}$ can be expressed as a mixture (3) provided that the number of components may be chosen sufficiently large. In particular, let $P(\boldsymbol{x})$ be a general discrete probability distribution on $\mathcal{X}$ defined by a table of probabilities. Considering a numbering of the points of $\mathcal{X}$, we can write

$$
\mathcal{X}=\cup_{k=1}^{K}\left\{\boldsymbol{x}^{(k)}\right\}, \quad P\left\{\boldsymbol{\xi}=\boldsymbol{x}^{(k)}\right\}=P\left(\boldsymbol{x}^{(k)}\right)=p^{(k)}, \quad \boldsymbol{x}^{(k)} \in \mathcal{X}, \quad k=1, \ldots, K
$$

where $p^{(k)}$ is the table probability attached to $\boldsymbol{x}^{(k)} \in \mathcal{X}$ and $K=|\mathcal{X}|$. Then the distribution mixture of the form (3) equivalent to the given table of values is obtained by setting

$$
w_{m}=p^{(m)}, \quad F(\boldsymbol{x} \mid m)=\prod_{n \in \mathcal{N}} \delta\left(x_{n}, x_{n}^{(m)}\right), \quad m=1, \ldots, K .
$$

In other words, the components $F(\boldsymbol{x} \mid m)$ in (9) are reduced to Dirac distributions $\delta\left(\boldsymbol{x}, \boldsymbol{x}^{(m)}\right)$ positioned at the points $\boldsymbol{x}^{(m)} \in \mathcal{X}$ while the component weights $w_{m}$ are equal to the respective table probabilities $p^{(m)}$.

\section{Problem of Identifiability}

The conditional independence model has been used by many authors in different areas as a tool of cluster analysis 18 . One of the most popular application fields appears to be the bacterial taxonomy. Gyllenberg et al. 12 recall about thirty references relating to a widely used method of classification of bacteria known as probabilistic numerical identification. The method is based on estimating parameters of the multivariate Bernoulli mixtures (4) from the observed data. The resulting components of the Bernoulli mixture are then used to identify the individual classes of bacteria (so called taxons). The posterior probability $q(m \mid \boldsymbol{x})$ 
(cf. (7)) is known in bacterial identification as the Willcox probability that the observed bacteria strain $\boldsymbol{x}$ belongs to the m-th class (taxon). In this sense the estimated Bernoulli mixture (4) defines the taxonomic structure of bacteria.

It is obvious that, before estimating the mixture (4), we should verify that it can be estimated uniquely, since otherwise we could obtain several different taxonomic structures for a given set of bacterial data. If the distribution mixture (3) or (44) is not defined uniquely then the corresponding interpretation of data in terms of clusters or latent classes becomes questionable (cf. [14, 12]). Unfortunately, multivariate Bernoulli mixtures are not identifiable, i.e. different parameter sets $\Theta=\left\{M, w_{m}, f_{n}(\cdot \mid m), m \in \mathcal{M}\right\}$ can correspond to exactly the same Bernoulli mixture.

Essentially, the proof of this assertion follows from the early papers of Teicher [17] and Blischke 2]. More recently Gyllenberg et al. 12 alternatively repeated the proof of Teicher for the specific case of discrete distributions by showing that the conditional independence model (3) is identifiable if and only if the mixtures of univariate discrete distributions $f_{n}\left(x_{n} \mid m\right)$ are identifiable. Then, by using a theorem of Blischke (cf. 2]), they show the mixtures of univariate Bernoulli distributions to be non-identifiable as a special case of the non-identifiable binomial distributions and therefore multivariate Bernoulli mixtures are non-identifiable, too. In the following we give a simple and intuitive proof of this property for a more general class of discrete mixtures with product components (cf. 9]).

Lemma 1. Any discrete distribution mixture of the form

$$
P(\boldsymbol{x})=\sum_{m \in \mathcal{M}} w_{m} F(\boldsymbol{x} \mid m), \quad F(\boldsymbol{x} \mid m)=\prod_{n \in \mathcal{N}} f_{n}\left(x_{n} \mid m\right), \quad \boldsymbol{x} \in \mathcal{X},\left(w_{m}>0\right) .
$$

can be equivalently described by infinitely many non-trivially different parameter sets $\Theta^{\prime}=\left\{M^{\prime}, w_{m}^{\prime}, f_{n}^{\prime}(\cdot \mid m), m \in \mathcal{M}^{\prime}\right\}$ if at least one of the univariate conditional distributions $f_{n}(\cdot \mid m)$ is non-singular in the sense that

$$
f_{n}\left(x_{n} \mid m\right)<1, \quad \text { for all } x_{n} \in \mathcal{X}_{n} .
$$

Proof. One can easily verify that any univariate discrete distribution $f_{n}(\cdot \mid m)$ which is non-degenerate in the sense of the inequality (11) can be expressed as a convex combination of two different distributions in infinitely many ways, e.g. $(0<\alpha<1, \quad \beta=1-\alpha)$ :

$$
f_{n}(\cdot \mid m)=\alpha f_{n}^{(\alpha)}(\cdot \mid m)+\beta f_{n}^{(\beta)}(\cdot \mid m), \quad f_{n}^{(\alpha)}(\cdot \mid m) \neq f_{n}^{(\beta)}(\cdot \mid m) .
$$

Now, by means of the substitution (12), we can express the component $w_{m} F(\boldsymbol{x} \mid m)$ as a weighted sum of two different components $F^{(\alpha)}(\boldsymbol{x} \mid m), F^{(\beta)}(\boldsymbol{x} \mid m)$

$$
w_{m} F(\boldsymbol{x} \mid m)=w_{m}^{(\alpha)} F^{(\alpha)}(\boldsymbol{x} \mid m)+w_{m}^{(\beta)} F^{(\beta)}(\boldsymbol{x} \mid m), \quad \boldsymbol{x} \in \mathcal{X}
$$

where

$$
w_{m}^{(\alpha)}=\alpha w_{m}, \quad F^{(\alpha)}(\boldsymbol{x} \mid m)=f_{n}^{(\alpha)}\left(x_{n} \mid m\right) \prod_{i \in \mathcal{N}, i \neq n} f_{i}\left(x_{i} \mid m\right),
$$


Table 1. Example of the 16-dimensional Bernoulli mixture from the paper [3]

\begin{tabular}{|c|c|c|c|c|c|c|c|c|c|c|c|c|c|c|c|c|}
\hline$w_{m}$ & $\vartheta_{1}$ & $\vartheta_{2}$ & $\vartheta_{3}$ & $\vartheta_{4}$ & $\vartheta_{5}$ & $\vartheta_{6}$ & $\vartheta_{7}$ & $\vartheta_{8}$ & $\vartheta_{9}$ & $\vartheta_{10}$ & $\vartheta_{11}$ & $\vartheta_{12}$ & $\vartheta_{13}$ & $\vartheta_{14}$ & $\vartheta_{15}$ & $\vartheta_{16}$ \\
\hline $0.222 \dot{2}$ & 0.80 & 0.80 & 0.80 & 0.80 & 0.20 & 0.20 & 0.20 & 0.20 & 0.20 & 0.20 & 0.20 & 0.20 & 0.20 & 0.20 & 0.20 & 0.20 \\
\hline 0.1944 & 0.20 & 0.20 & 0.20 & 0.20 & 0.80 & 0.80 & 0.80 & 0.80 & 0.20 & 0.20 & 0.20 & 0.20 & 0.20 & 0.20 & 0.20 & 0.20 \\
\hline 0.1666 & 0.20 & 0.20 & 0.20 & 0.20 & 0.20 & 0.20 & 0.20 & 0.20 & 0.80 & 0.80 & 0.80 & 0.80 & 0.20 & 0.20 & 0.20 & 0.20 \\
\hline $0.138 \dot{8}$ & 0.20 & 0.20 & 0.20 & 0.20 & 0.20 & 0.20 & 0.20 & 0.20 & 0.20 & 0.20 & 0.20 & 0.20 & 0.80 & 0.80 & 0.80 & 0.80 \\
\hline $0.111 \dot{1}$ & 0.80 & 0.20 & 0.20 & 0.20 & 0.80 & 0.20 & 0.20 & 0.20 & 0.80 & 0.20 & 0.20 & 0.20 & 0.80 & 0.20 & 0.20 & 0.20 \\
\hline $0.083 \dot{3}$ & 0.20 & 0.80 & 0.20 & 0.20 & 0.20 & 0.80 & 0.20 & 0.20 & 0.20 & 0.80 & 0.20 & 0.20 & 0.20 & 0.80 & 0.20 & 0.20 \\
\hline $0.055 \dot{5}$ & 0.20 & 0.20 & 0.80 & 0.20 & 0.20 & 0.20 & 0.80 & 0.20 & 0.20 & 0.20 & 0.80 & 0.20 & 0.20 & 0.20 & 0.80 & 0.20 \\
\hline $0.027 \dot{7}$ & 0.20 & 0.20 & 0.20 & 0.80 & 0.20 & 0.20 & 0.20 & 0.80 & 0.20 & 0.20 & 0.20 & 0.80 & 0.20 & 0.20 & 0.20 & 0.80 \\
\hline
\end{tabular}

$$
w_{m}^{(\beta)}=\beta w_{m}, \quad F^{(\beta)}(\boldsymbol{x} \mid m)=f_{n}^{(\beta)}\left(x_{n} \mid m\right) \prod_{i \in \mathcal{N}, i \neq n} f_{i}\left(x_{i} \mid m\right)
$$

and therefore, after substitution (13) in (10), we obtain two formally different mixtures defined by different parameter sets $\Theta \neq \Theta^{\prime}$ which describe exactly the same probability distribution $P(\boldsymbol{x})$.

It can be seen that the non-identifiability of any non-degenerate Bernoulli mixture (4) directly follows from Lemma 1. It appears that the question of uniqueness has been neglected in the literature on numerical taxonomy (cf. discussion in [12]) but, surprisingly, this circumstance does not seem to have any serious practical consequences. Moreover, it has been observed that in numerical experiments the mixture parameters can often be uniquely identified from sufficiently large randomly generated samples of data vectors.

Thus e.g. Carreira-Perpinan et al. 3] in a re-identification experiment generated randomly a set of 10000 of 16 dimensional binary vectors from a specific Bernoulli mixture of $M=8$ components (cf. Tab. 1). Using this data they estimated repeatedly Bernoulli mixtures of different number of components by means of EM algorithm. For $M=8$ the original parameters were re-identified 9 out of 10 times. When using fewer components $(M=4)$ the EM algorithm reproduced some of the original components and linear combinations of the remaining ones. A more complex mixture model $(M=10)$ always reproduced the eight original components with the last two being their slight modifications or linear combinations. We have observed similar results in our early paper 7]. It appears that "well separated" components in high-dimensional spaces are "practically identifiable" (cf. [3]) if the data set $\mathcal{S}$ is large enough.

\section{Sequential Identification of Components}

For obvious reasons the theoretically possible ambiguity in estimating discrete models of conditional independence is a serious disadvantage from the point of view of practical applications. Nevertheless, we can achieve a unique result of cluster analysis e.g. by introducing additional constraints. One intuitively 
Table 2. Parameters of the 16-dimensional Bernoulli mixture obtained by re-estimating the mixture parameters from Tab.1 by using sequential adding of components

\begin{tabular}{|c|c|c|c|c|c|c|c|c|c|c|c|c|c|c|c|c|}
\hline$w_{m}$ & $\vartheta_{1}$ & $\vartheta_{2}$ & $\vartheta_{3}$ & $\vartheta_{4}$ & $\vartheta_{5}$ & $\vartheta_{6}$ & $\vartheta_{7}$ & $\vartheta_{8}$ & $\vartheta_{9}$ & $\vartheta_{10}$ & $\vartheta_{11}$ & $\vartheta_{12}$ & $\vartheta_{13}$ & $\vartheta_{14}$ & $\vartheta_{15}$ & $\vartheta_{16}$ \\
\hline .2220 & .800 & .800 & .800 & .800 & .200 & .200 & .200 & .200 & .200 & .200 & .200 & .200 & .200 & .200 & .200 & .200 \\
\hline .1943 & .200 & .200 & .200 & .200 & .800 & .800 & .800 & .800 & .200 & .200 & .200 & .200 & .200 & .200 & .200 & .200 \\
\hline .1666 & .200 & .200 & .200 & .200 & .200 & .200 & .200 & .200 & .800 & .800 & .800 & .800 & .200 & .200 & .200 & .200 \\
\hline .1388 & .200 & .200 & .200 & .200 & .200 & .200 & .200 & .200 & .200 & .200 & .200 & .200 & .800 & .800 & .800 & .800 \\
\hline .1109 & .800 & .200 & .200 & .200 & .800 & .200 & .200 & .200 & .800 & .200 & .200 & .200 & .800 & .200 & .200 & .200 \\
\hline .0832 & .200 & .800 & .200 & .200 & .200 & .800 & .200 & .200 & .200 & .800 & .200 & .200 & .200 & .800 & .200 & .200 \\
\hline .0555 & .200 & .200 & .800 & .200 & .200 & .200 & .800 & .200 & .200 & .200 & .800 & .200 & .200 & .200 & .800 & .200 \\
\hline .0277 & .200 & .200 & .200 & .800 & .200 & .200 & .200 & .800 & .200 & .200 & .200 & .800 & .200 & .200 & .200 & .800 \\
\hline .0008 & .442 & .392 & .371 & .355 & .395 & .348 & .327 & .312 & .373 & .327 & .307 & .292 & .359 & .314 & .294 & .280 \\
\hline
\end{tabular}

acceptable and easy to apply method is a sequential adding of new components to the estimated mixture.

In particular, applying EM algorithm, we start with a mixture having a single component and arbitrary (e.g. randomly chosen) initial parameters. In this case the EM algorithm converges in one iteration to a component defined as a product of univariate marginal distributions. In the next phase a new component is added, initialized as a product of univariate uniform distributions with equal initial weight, i.e. $w_{1}=w_{2}=0.5$. Then the EM iterations are continued until sufficient convergence. When the relative increase of the likelihood function is less then some small positive threshold $\epsilon$, a new uniform component is added again and the component weights are normed to obtain $w_{3}=w_{2}$ and $w_{1}+w_{2}+w_{3}=1$. The EM iterations are then started again with the new initial parameters. In this way the new component defined as a product of uniform marginals is added repeatedly as long as it is "accepted" by the previous mixture model. The computation is stopped when the weight of the new added component is less than a suitably chosen low threshold after the convergence is achieved.

There is no theoretical support of the proposed method to guarantee some qualitative properties of the resulting mixture. Nevertheless, it can be heuristically justified by some computational properties. Let us note first that, from the computational point of view, the resulting mixture model is defined uniquely. In practice, the only source of uncertainty may be the limited parameter accuracy at the end of each convergence phase. Moreover, the method avoids random influences of initial values and represents a reasonable mechanism to choose a proper number of components. Note that the newly added uniform component tends to "fit" to data points insufficiently "covered" by the previous model and for this reason the weight of the added component is usually decreasing in final stages of computation when the number of components is sufficiently large.

It should be emphasized that by including a new (uniform) component to the previously adjusted model we may violate the monotonic property of EM algorithm in the following iteration. Moreover the new component interferes with the previously estimated parameters and partly devaluates the preceding convergence phase. 
We illustrate the proposed sequential identification method by considering the re-identification problem from the paper [3. In order to avoid random influences of a given finite sample $\mathcal{S}$ we re-estimated the original multivariate Bernoulli mixture (cf. Tab.1) in a way which is equivalent to an infinite sample size. Note that for the sample size $|\mathcal{S}|$ approaching infinity we can write

$$
L^{*}=\lim _{|\mathcal{S}| \rightarrow \infty} \frac{1}{|\mathcal{S}|} \sum_{x \in \mathcal{S}} \log \left[\sum_{m \in \mathcal{M}} w_{m} F(\boldsymbol{x} \mid m)\right]=\sum_{x \in \mathcal{X}} P^{*}(\boldsymbol{x}) \log \left[\sum_{m \in \mathcal{M}} w_{m} F(\boldsymbol{x} \mid m)\right]
$$

i.e. the sum over the infinite sample $\mathcal{S}$ can be equivalently replaced by summing over all $\boldsymbol{x} \in \mathcal{X}$ whereby $P^{*}(\boldsymbol{x})$ denotes the asymptotic relative frequency of $\boldsymbol{x}$.

In order to maximize the asymptotic likelihood function $L^{*}$ we have modified the basic EM iteration Eqs. (7), (8) in analogy with Eq. (14):

$$
\begin{gathered}
w_{m}^{\prime}=\sum_{x \in \mathcal{X}} P^{*}(\boldsymbol{x}) q(m \mid \boldsymbol{x}), \quad m \in \mathcal{M}, \\
\vartheta_{n m}^{\prime}=\frac{1}{\sum_{x \in \mathcal{X}} P^{*}(\boldsymbol{x}) q(m \mid \boldsymbol{x})} \sum_{x \in \mathcal{X}} x_{n} P^{*}(\boldsymbol{x}) q(m \mid \boldsymbol{x}), \quad n \in \mathcal{N} .
\end{gathered}
$$

Instead of generating a given number of pseudo-random binary vectors we have computed and stored the values $P^{*}(\boldsymbol{x})$ for all the 65536 binary vectors $\boldsymbol{x}$ from the 16-dimensional binary cube. By using the "asymptotic" likelihood function $L^{*}$ and the corresponding version of EM algorithm we have the possibility to avoid any random small sample fluctuations. In other words we can verify the properties of the proposed method in the extreme case of infinite sample size.

The method of sequential adding of components based on the asymptotically modified EM iteration equations (15), (16) has been applied repeatedly to reestimate the mixture parameters from Tab.1. A new component has been added whenever the relative increase of the maximized criterion $L^{*}$ was less than a chosen threshold $\epsilon=10^{-12}$. The estimated parameters from Tab.2 have been obtained after 3000 iterations. The threshold $\epsilon$ has been varied between $10^{-9}$ and $10^{-12}$ with very similar results. In all computational experiments we have observed a clear tendency to suppress the weight of superfluous components. The 10 th component was not added and the weight of the last added 9 th component was by two or three orders less than $w_{8}$, i.e. $w_{9} \approx 10^{-4}-10^{-5}$ (cf. Tab. 2).

\section{Conclusion}

The models of conditional independence have been proposed repeatedly as a tool of cluster analysis of multivariate categorical data since the standard approaches are usually not directly applicable. A serious drawback of the conditional independence models follows from the fact that they are not uniquely identifiable. We give a simple and intuitive proof that any non-degenerate discrete mixture with product components can be equivalently described by infinitely many different parameter sets and therefore it is non-identifiable. We propose to guarantee a 
unique result of cluster analysis by introducing additional constraints, in particular by sequential adding of components in EM algorithm.

Let us recall finally that there are numerous application possibilities of the conditional independence models based on approximating unknown probability distributions (cf. e.g. 7], 8], [10, [11). In application to practical problems of pattern recognition and statistical modelling the approximation accuracy is of primary importance. The non-identifiability of estimated mixtures is less relevant and may be even useful in view of increased flexibility of mixture models.

\section{References}

1. Bartholomew D.J.: Factor analysis for categorical data. J. Roy. Statist. Soc. B, 3 42 (1980) 293-321

2. Blischke W.R. : Estimating the parameters of mixtures of binomial distributions. Journal Amer. Statist. Assoc., 59 (1964) 510-528

3. Carreira-Perpignan M.A., Renals S.: Practical identifiability of finite mixtures of multivariate Bernoulli distributions. Neural Computation, 12 (2000) 141-152

4. Dempster, A.P., Laird, N.M. and Rubin, D.B.: Maximum likelihood from incomplete data via the EM algorithm. J. Roy. Statist. Soc. B, 39 (1977) l-38

5. Fielding A.: Latent structure models. In: The Analysis of survey data, (Eds. O'Muircheaxtaigh C.A, Payne C.), London: Wiley, (1977) 125-157

6. Gibson W.A.: Three multivariate models: Factor analysis, latent structure analysis and latent profile analysis. Psychometrika, 24 (1969) 229-252

7. Grim J.: Multivariate statistical pattern recognition with nonreduced dimensionality, Kybernetika, 22 (1986) 142-157

8. Grim J., Boček P., Pudil P. (2001): Safe dissemination of census results by means of interactive probabilistic models. In: Proceedings of the ETK-NTTS 2001 Conference, (Hersonissos (Crete), European Communities 2001, 2 (2001) 849-856

9. Grim J.: Latent Structure Analysis for Categorical Data. Research Report UTIA, No. 2019, 13 pp., Academy of Sciences, Czech Republic, Prague (2001)

10. Grim J., Haindl M.: Texture Modelling by Discrete Distribution Mixtures. Computational Statistics and Data Analysis, 3-4 41 (2003) 603-615

11. Grim J., Kittler J., Pudil P., Somol P.: Multiple classifier fusion in probabilistic neural networks. Pattern Analysis \& Applications, 75 (2002) 221-233

12. Gyllenberg M., Koski T., Reilink E., Verlaan M.: Non-uniqueness in probabilistic numerical identification of bacteria. Journal of Applied Prob., 31 (1994) 542-548

13. McLachlan G.J. and Peel D.: Finite Mixture Models, John Wiley \& Sons, New York, Toronto, (2000)

14. Lazarsfeld P.F., Henry N.: Latent structure analysis. Houghton Mifl.: Boston (1968)

15. Pearl J.: Probabilistic reasoning in intelligence systems: networks of plausible inference. Morgan-Kaufman, San Mateo, CA (1988)

16. Suppes, P.A.: Probabilistic theory of causality. North-Holland: Amsterdam, (1970)

17. Teicher, H. : Identifiability of mixtures of product measures. Ann. Math. Statist., 39 (1968) 1300-1302

18. Vermunt J.K., Magidson J.: Latent Class Cluster Analysis. In: Advances in Latent Class Analysis, (Eds. Hagenaars J.A. et al.), Cambridge Univ. Press (2002) 